\title{
BMJ Open Protocol for the Lovisenberg Open Acute Door Study (LOADS): a pragmatic randomised controlled trial to compare safety and coercion between open-door policy and usual-care services in acute psychiatric inpatients
}

Nikolaj Kunøe (D) , Hans Martin Nussle, Anne-Marthe Indregard

To cite: Kunøe N, Nussle HM, Indregard A-M. Protocol for the Lovisenberg Open Acute Door Study (LOADS): a pragmatic randomised controlled trial to compare safety and coercion between open-door policy and usual-care services in acute psychiatric inpatients. BMJ Open 2022;12:e058501. doi:10.1136/ bmjopen-2021-058501

- Prepublication history for this paper is available online. To view these files, please visit the journal online (http://dx.doi org/10.1136/bmjopen-2021058501).

Received 19 0ctober 2021 Accepted 14 January 2022
Check for updates

(C) Author(s) (or their employer(s)) 2022. Re-use permitted under CC BY-NC. No commercial re-use. See rights and permissions. Published by BMJ.

Department of Psychiatry, Lovisenberg Diakonale Sykehus AS, 0slo, Norway

Correspondence to

Dr Nikolaj Kunøe; nku@lds.no

\section{ABSTRACT}

Introduction The reduction of coercion in psychiatry is a high priority for both the WHO and many member countries. Open-door policy (ODP) is a service model for psychiatric ward treatment that prioritises collaborative and motivational measures to better achieve acute psychiatric safety - and treatment objectives. Keeping the ward main door open is one such measure. Evidence on the impact of ODP on coercion and violent events is mixed, and only one randomised controlled trial (RCT) has previously compared ODP to standard practice. The main objectives of the Lovisenberg Open Acute Door Study (LOADS) are to implement and evaluate a Nordic version of ODP for acute psychiatric inpatient services. The evaluation is designed as a pragmatic RCT with treatment-as-usual (TAU) control followed by a 4-year observational period.

Methods and analysis In this 12-month pragmatic randomised trial, all patients referred to acute ward care will be randomly allocated to either TAU or ODP wards. The primary outcome is the proportion of patient stays with one or more coercive measures. Secondary outcomes include adverse events involving patients and/or staff, substance use and users experiences of the treatment environment and of coercion. The main hypothesis is that ODP services will not be inferior to state-of-the art psychiatric treatment. ODP and TAU wards are determined via ward-level randomisation. Following conclusion of the RCT, a longitudinal observational phase begins designed to monitor any long-term effects of ODP.

Ethics and dissemination The trial has been approved by the Regional Committees for Medical and Health Research Ethics (REC) in Norway (REC South East \#29238), who granted LOADS exemption from consent requirements for all eligible, admitted patients. Data are considered highly sensitive but can be made available on request. Results will be published in peer-reviewed journals and presented at scientific conferences and meetings.

Trial registration number ISRCTN16876467.

Protocol version 1.4, 21 December 2021.

\section{INTRODUCTION}

Balancing restrictive and collaborative measures in ward-based psychiatric treatment
Strengths and limitations of this study

- Protocol enhancing accountability and replication of a potentially coercion-reducing service model in acute psychiatric wards.

- The planned study will be the first randomised controlled trial on open-door policy in psychiatry in 25 years.

- Generalisability is enhanced due to ethical committee permission to include all eligible patients with exemption from ordinary consent rules.

- Weaknesses include vulnerability to historic and community events, a lack of standardised fidelity checklists for the intervention and the inability to mask allocated groups.

has been a topic of discussion since the early asylums. ${ }^{1}{ }^{2}$ Overly restrictive practices can provoke patients to resist treatment or cause post-traumatic stress, whereas relying too much on collaborative measures risks treatment drop-out and/or insufficient attentiveness to prevent patients from causing grave harm to themselves or others. As part of this debate, the WHO has called for member states to replace coercive practices with collaborative practices entirely, ${ }^{3}$ while the Lancet World Psychiatric Association commission on the Future of Psychiatry ${ }^{4}$ has called for more and better evidence on coercive practices.

One part of this discussion concerns whether routinely locking the main doors to psychiatric wards counteracts psychiatric healthcare's therapeutic mandate and influences the attitudes of staff and general society towards psychiatric inpatients. ${ }^{156}$ Since the early 2000's, researchers from hospitals in Germany and Switzerland have reported 
success in reducing coercive practices by introducing 'open-door policy' (ODP).

An ODP in a psychiatric ward could reduce coercion in several ways, for example:

1. The open ward door motivates staff to rely more on their ability to engage patients in collaborative and therapeutic activities to keep patients on the ward than would be the case if the door was locked. Such collaborative attitudes and practices constitute a large part of the 'policy' component of ODP. ${ }^{78}$

2. The open door itself emphasises the ward's potential as a place of treatment and help for the patient, counteracting some of the stigma towards psychiatric wards as places of confinement to the benefit of other members of society. Not locking doors by default also communicates understanding that patients' fundamental rights to freedom provide a basis for building or maintaining a long-term therapeutic collaboration with healthcare services.

3. Patients' opportunity to exit the ward through the open door may counteract factors such as crowding or provocation by fellow patients associated with increased risk of in-ward violent events and coercive measures.

4. The open door could increase the opportunity for contact with family, friends and community health services.

ODP shares characteristics and practices with several other user-centred models of ward-based psychiatry such as collaborative staff attitudes, integrated treatment of dual disorders, communication skills training, violence risk assessment, violent event prevention and de-escalation and suicide risk assessment. ${ }^{9-11}$ Peer-support workers are also frequently present on ODP wards to facilitate a therapeutic dialogue between staff and patients. ${ }^{71213}$

Several observational studies of open-door policy psychiatric wards suggest considerable potential for reductions in the use of coercive measures when compared with traditional, closed-door services. ${ }^{14-18}$ These results reflect the generally positive results from the only known randomised controlled trial (RCT) on the topic, conducted in China over 25 years ago. ${ }^{19}$ Yet some attempts to replicate $\mathrm{ODP}^{20-22}$ have resulted in mixed or mildly negative results. In addition, ODP can be criticised for a lack of rigour and a lack of fidelity checklists, ${ }^{5}$ as the ODP literature ${ }^{7}$ permits hospitals some freedom of choice in which elements of ODP to adopt in order to facilitate implementation.

\section{Objectives}

The Lovisenberg Open Acute Door Study (LOADS) is a pragmatic RCT $^{23}$ of an ODP for acute psychiatric inpatient services adapted to a Nordic healthcare service model. The impact of ODP on outcomes will be measured using a hybrid randomised design featuring: (1) a 12-month patient-level RCT of acute psychiatric patients in ODP versus treatment-as-usual (TAU) services and (2) a wardlevel 'stepped-wedge' randomised comparison of ODP and usual-care outcomes by comparing observations of
pre-RCT (no wards ODP) to during-RCT (two out of five wards ODP) and post-RCT periods (five wards ODP) with planned follow-up milestones at 12, 24, 36 and 48 months.

This protocol article describes the scientific rationales-and procedures for design, ethical conduct, data collection and-analysis. LOADS' primary hypothesis is that an ODP psychiatric acute ward service model is noninferior to or better than a usual-care psychiatric acute ward service model.

\section{METHODS}

This protocol conforms to the recommendations outlined in the Standard Protocol Items: Recommendations for Interventional Trials statement guidelines. ${ }^{24}$

\section{Study setting}

Psychiatric services in Norway are based on a universal healthcare model with three main service levels: community services include general physicians and municipal/ borough sponsored services such as emergency rooms, hospital-owned outpatient-and outreach services, and ward-based hospital services. Acute psychiatric wards manage cases and disorders assessed too difficult or too dangerous to manage in the other settings. Unlike most other countries, Norway does not have dedicated forensic psychiatric units, but high security wards exist that admit patients with a record of causing serious injury or death.

Legally sanctioned coercive measures in Norway include involuntary medication (acute tranquillising or sedative medication and long-term antipsychotic), seclusion/isolation, mechanical and physical restraints, involuntary observation and-medical examination. A preferred alternative to seclusion/isolation is accommodating patient in a separate part of the ward or building with fewer other patients and increased staff per patient called 'shielding' (in Norwegian: 'skjerming') ${ }^{25}$ All coercive measures including 'shielding must be authorised by a senior physician or psychologist specialised in adult psychiatry, and patients must be monitored regularly by staff. Coercive measures are only to be used once all options for voluntary measures have been exhausted, and coercion must only be utilised as briefly and humane as possible. Norway's mental health act permits community treatment orders, that are usually enacted following involuntary acute psychiatric admissions.

LOADS is conducted on a single study site, the Lovisenberg Diaconal Hospital (LDH). The LDH catchment area includes three inner-city boroughs in Oslo, Norway: St. Hanshaugen, Grünerløkka, Gamle Oslo. In addition, around $12 \%$ of patients are admitted from surrounding boroughs. The catchment area for acute psychiatric admissions includes a population of around 270000 inhabitants. This area includes Oslo's main railway station and the majority of the city's open drug scenes, areas that have Norway's highest per capita levels of social - and mental health problems. 
On the LDH Campus, one high-rise building the 'L21F' houses all wards belonging to the LDH Department of Psychiatry. The six floors of the L21F includes a psychiatric admissions ward/psychiatric intensive care unit (PICU) on the ground floor, and five regular wards with ten beds each located on the floors above. In addition to the five regular wards, patients with a need for extraordinary resources during treatment, for example due to extra high risk of aggression or violence, can be referred to an eight-bed ward with more staff and less crowding. Patients treated on this high-resource ward are exempt from randomisation in this trial, but may be described in LOADS disseminations on a group basis.

During a typical year the LDH Department of Psychiatry receives approximately 800 referred patients to 1100 acute admissions, of which around $40 \%$ are brief stays at the PICU/admissions ward. Patients in need of further acute ward treatment are transferred to one of five regular acute wards situated on the upper floors of the building where they stay for an average of 18 days. On these wards, around two-thirds of patients are involuntarily admitted, and a similar proportion have psychotic disorders (bipolar - or schizophrenia disorders).

Patients can engage in music therapy, physical activity, art therapy, work therapy skills training, and sensory stimulation rooms. Except for a small rooftop terrace, gardens and other outdoor facilities can only be accessed by leaving the building via the ground floor. Staffing factor (full-time equivalents) is approximately two patients per nursing staff during daytime and 3-4 patients per staff during night-time.

\section{Patient and public involvement}

The LDH Board of service users has been actively involved in LOADS since early planning stages (including feasibility evaluation), as well as discussions of study design including choice of outcome measures and evaluation of the ethics of participating in the study. The LDH Board of service users is represented in the steering group overseeing implementation of LOADS and will be involved in interpretation of the data.

\section{Study design}

The LOADS evaluation study is a mixed design pragmatic, randomised controlled non-inferiority trial ${ }^{23}$ combined with a ward-level observational study of longterm outcomes.

\section{Participants and procedures}

All admitted adult patients (18 years old or older) referred to acute psychiatric ward care at the LDH are eligible to participate. The majority of eligible patients undergo 1-2 days of risk assessment and eligibility screening on the admission ward, while patients from outside the catchment area (12\% of patients in 2020) have undergone this screening elsewhere.

On referral to ward care in LOADS, patients are allocated by clinical staff to either ODP or regular acute psychiatric ward services using a simple $2 / 5$ vs $3 / 5$ random distribution list generated on random.org

Patients evaluated to need extraordinary staffing resources for example due to high and imminent risk of violent behaviour, are considered non-eligible and referred to a single high-resource acute ward.

\section{Ward (stepped-wedge) randomisation}

As the admissions ward and the high-resource ward perform non-standard care, there are five wards eligible for ward-level randomisation. Two of five wards are randomly allocated ${ }^{26}$ to commence implementing ODP to their allocated patients (see figure 1 and section on patient randomisation below) starting February 2021. After 12 months, effects of ODP will be evaluated using clinical outcome monitoring. If ODP appears to improve or is not inferior to TAU, additional wards in the building will be randomly allocated to commence implementing ODP from month 13 onwards.

\section{Patient randomisation}

Any participants referred from the admissions ward to standard ward care will be randomly allocated to either the two ODP wards or three standard policy wards.

Allocation to study arms will take place in a chronological, 'trickle design', using a simple, numbered, binomial

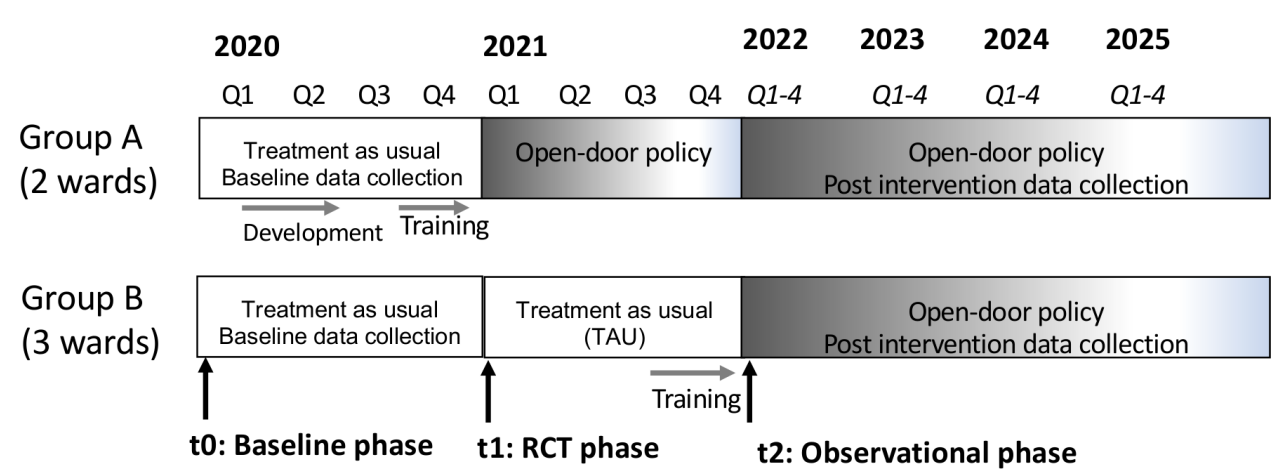

Figure 1 The Lovisenberg Open Acute Door Study (LOADS) is stepped-wedge pre-post design that consists of a 12-month baseline phase where intervention and usual-treatment wards are randomly selected, continues with a 12-month patient-level intervention versus usual-treatment RCT, and a 4-year longitudinal observational follow-up during which all wards implement the intervention. RCT, randomised controlled trial. 
list for continuous randomisation to either ODP or usual treatment wards. The sequence was generated by the Principal Investigator (PI) on random.org and reflects the number of wards in each condition: $2 / 5$ of the numbers are allocated to ODP and $3 / 5$ to ordinary policy wards. The allocation will be administered by admission staff in a group setting during day shifts and evening shifts, and by the admissions ward head nurse during nightshifts. Random allocation and study arm is open label to staff, researchers (including PI) and patients as masking of ward service models is not considered feasible due to the significance of both staff and patients. Adherence to randomisation procedures is verified by LOADS management every month, and any discrepancies are discussed with the admissions staff.

As a healthcare services study conducted within the confines of the LDHs acute legal and medical responsibilities for referred patients, LOADS cannot pause inclusion due to scientific reasons, for example, if all beds in one or both study arms are full. If such situations occur, admission staff is instructed to preserve the integrity of the study by allocating patients according to principles not related to hypotheses or rationale of ODP versus usual care by placing patients in either (1) the first bed available or, (2) if all beds on all wards are full, to any available bed on any of the wards.

To ensure continuity of treatment for patients in the event of readmission, patients will only be randomised once during the trial period, after which allocation will follow the 'home ward' principle of admission to the ward or policy in which originally allocated.

Patients with a court order of confinement or a history of repeated aggression during psychiatric treatment will be exempt from randomisation and instead admitted to the eight-bed, high-resource ward with extra staff. Descriptive data on this patient group will be compared with that of allocated patients to ensure transparency on criteria for treatment.

\section{Intervention}

The ODP service procedures implemented at LDH have been developed by staff and service users based on visits to wards in Basel and Berlin, as well as descriptions of clinical practice with ODP in the German-language and English-language literature. $^{727}$

The main doors to the wards are open from 9 to 21 hours all days of the week but can be closed in case of emergency ('facultative' or 'partial' opening). While the door mechanism permits patients to freely exit the ward, re-entry into the ward requires active assistance by staff after the patient rings the doorbell.

The 'policy' component is a staff attitude component designed to build trust and collaboration between patients and staff, and ultimately between patient and treatment services. This component includes several practices that are not unique to ODP but may be easier to perform systematically within the ODP than within the usual healthcare service framework. For example, empowerment of admitted patients is encouraged by repeated attempts at involvement in activities and treatment and offer of contracts for self-referral to frequently readmitted patients. Emphasis is placed on collaboration through shared decision making. Network meetings with the patient and any third parties to facilitate communication and collaboration. Priority is given to interventions and activities that can support longer-term recovery over short-term symptomatic relief.

\section{TAU/control group}

On TAU wards, patients' freedom of mobility out of the ward will depend on staffs' availability and individual risk assessment. Usual-treatment wards have not specified their attitude/"policy' towards patients but remain part of a culture at the LDH that has previously received honorable mention for its coercion reduction efforts. ${ }^{28}$ Staff on all wards in the study undergo training in de-escalation and harm-minimisation every 3-6 months.

\section{Study outcomes}

LOADS outcomes have been selected for comparability with previous findings on ODP psychiatry in Germany, Switzerland and elsewhere. Due to the limited consensus on clinically significant differences (vs non-significant) in acute psychiatric outcomes, the level of least clinically significant difference for all measures has been set to $15 \%$ for calculation of non-inferiority and superiority. Table 1 provides an overview of LOADS' primary and secondary outcomes.

\section{Primary outcome}

The LOADS primary outcome is the proportion of patient stays with one or more coercive measures. This outcome includes all instances of seclusion, isolation, involuntary medication, physical and mechanical restraints towards patients admitted to any of the five participating wards from study start in February 2021 to the end of the 12-month patient-level RCT in February 2022. After the RCT and the trial progresses in a longitudinal observational design, this variable will be included in the annual summary of data at 24, 36 and 48 months.

\section{Secondary outcomes}

Patient feedback is standard at Lovisenberg wards and currently include the Essen Climate Evaluation Scale ${ }^{29}$ (EssenCES) and perception of coercion measured using the Experience of Coercion Scale. ${ }^{30}$ The EssenCES can also be scored by staff for a separate perspective on ward environment. Overall levels of substance use will be measured by chromatographic analysis of illicit substances and their metabolites in samples from the building's wastewater.

Outcomes from different types of coercive measures or types of adverse events may be reported separately and subject to in-depth analyses in separate publications.

The amount of time ward main doors and side-wing or 'skjerming' doors were open or closed is recorded by ward staff are secondary variables that may be associated 
Table 1 Primary and secondary outcomes in the Lovisenberg Open Acute Door Study

\begin{tabular}{|c|c|c|c|c|}
\hline Primary outcome & Variable type & Data source & Hypothesis RCT & Hypothesis pre-post \\
\hline Proportion of admissions $w / 1+$ coercive measures & Event (Nominal) & $\begin{array}{l}\text { Hospital } \\
\text { records }\end{array}$ & Non-inferiority & Reduction (superiority) \\
\hline Secondary outcomes & Variable type & Data source & Hypotheses RCT & $\begin{array}{l}\text { Hypotheses pre- } \\
\text { post }\end{array}$ \\
\hline $\begin{array}{l}\text { Violent events such as violence against staff or completed } \\
\text { suicides }\end{array}$ & Event (Nominal) & $\begin{array}{l}\text { Hospital } \\
\text { records }\end{array}$ & Non-inferiority & Reduction (superiority) \\
\hline Intensive care or 'skjerming' & Event & $\begin{array}{l}\text { Hospital } \\
\text { records }\end{array}$ & Non-inferiority & Reduction (superiority) \\
\hline Duration of coercive measure $(\mathrm{s})^{\star}$ & Continuous & $\begin{array}{l}\text { Hospital } \\
\text { records }\end{array}$ & Non-inferiority & Reduction (superiority) \\
\hline Absconding or unplanned absence from ward care & $\begin{array}{l}\text { Event (nominal) } \\
\text { and duration } \\
\text { of absence } \\
\text { (continuous) }\end{array}$ & $\begin{array}{l}\text { Hospital } \\
\text { records }\end{array}$ & Non-inferiority & Non-inferiority \\
\hline
\end{tabular}

${ }^{*}$ Comparisons on the duration of coercive measures is contingent on records meeting minimum requirements for data quality to permit statistical analysis

$\mathrm{RCT}$, randomised controlled trial.

with other outcomes in secondary analyses. Absconding is included due to interest in previous publications, even if its relevance to clinical practice in low-risk patients is controversial.

Frequently used measures of acute psychiatric ward treatment such as readmission rates, length of stay, time to readmission will be included in analyses as appropriate.

The presence or availability of ODP-related services such as peer support workers or activities such as opendialogue style meetings may also be registered and subject to separate analyses.

Serious adverse events like completed suicides or violence against staff occur with regular intervals in acute psychiatric treatment. Completed suicides on the LDH grounds occur at a rate of 1-4 events per year, making them difficult to analyse statistically.

Non-inferiority hypotheses were chosen based on (1) the mixed results reported in previous studies and (2) because a less restrictive approach to patient freedom of mobility is a beneficial result even if coercive measures and adverse events go unchanged and (3) unlike previous comparative publications, the LOADS' 'usual treatment' has received recognition for achieving coercion prevention and reduction. ${ }^{28}$ In addition, there is uncertainty regarding whether 12 months will be sufficient for a complex intervention like ODP to take effect; while one report from Berlin suggests effects at 6 months, ${ }^{31}$ other studies have not reported significant effects on different outcomes until ODP has been implemented for much longer, for example, 24-48 months. ${ }^{32} 33$

Background data will be reported in order to provide readers with information on the social and material context of the main outcomes, such as patient or staff demographics. Examples of these data are provided in table 2.

\section{Stop rules}

The LOADS steering group is authorised to halt the trial on safety reasons or 'stop rules' should an extraordinary accumulation of events occur during a short period of

Table 2 Background variables and other variables of interest in the Lovisenberg Open Acute Door Study

\begin{tabular}{|c|c|}
\hline Variable type & Data source \\
\hline $\begin{array}{l}\text { Patient-level (demographics): age, biological gender, admitted involuntarily, assessed violence risk at } \\
\text { admission, readmission, admissions via self-referral }\end{array}$ & Hospital records \\
\hline $\begin{array}{l}\text { Ward-level: Duration of stay, time to readmission, days until readmissions, proportion of opening hours } \\
\text { closed, extra staff resources used }\end{array}$ & Hospital and ward records \\
\hline $\begin{array}{l}\text { Workplace-related: Staff perception of ward climate and staff perception of workplace safety, } \\
\text { wastewater levels of substances of abuse and /or - metabolites }\end{array}$ & $\begin{array}{l}\text { Hospital and ward records, building- } \\
\text { wide wastewater analysis }\end{array}$ \\
\hline
\end{tabular}


time that could reasonably be attributed to ODP ('stop rules'):

- Four or more completed suicides within a 1-month period (corresponding to a full year's prevalence of suicides on hospital premises).

- More than a $100 \%$ increase in the month-by-month number of violent incidents against ward staff.

- More than a $100 \%$ increase in the month-by-month total number of reported serious adverse events (violent events, suicides, involving care of multiple patients).

\section{Sample size}

Clinical data on the number of patients subjected to coercive measures in 2019 and 2020 have been used to determine sample size. The predefined least clinically significant difference of $15 \%$ and a significance level of $95 \%$ was entered into a chi square analysis of noninferiority on the powerandsamplesize.com website.

With a proportion of patients subjected to coercion in the LDH acute psychiatric wards corresponding to 0.33 vs 0.34 , the required sample size for determining noninferiority is a minimum of $\mathrm{n}=200$ in each group for a $90 \%$ power level ('beta') and $n=185$ with a beta level of $80 \%$. As each participating ward in 2020 treated approximately $\mathrm{n}=130$ patients, a 12 -month inclusion period in 2021 should enable LOADS to include approximately $\mathrm{n}=260$ patients on two wards in the ODP group and $\mathrm{n}=390$ patients from three wards in the usual-care group.

\section{Study organisation}

As LOADS is a healthcare services study based on clinical outcomes from ordinary acute psychiatric wards, the clinic director and head physician maintain their usual medical responsibility for patients during the study period. Research is organised as a research group headed by NK as the PI, with A-MI employed as senior researcher and HM acting as co-ordinator as part of his role as director of Research and Development. The steering committee meets for major milestones and critical decisions and includes a service user representative from the LDH Board of Service users, the research group including the PI, the clinic director, a representative of LDH Outreach specialist services, and Ward Managers. A Project group consisting of the Ward Managers from ODP wards meets once a month for calibration of their wards' implementation of ODP. A Reference group meets annually and consists of the Steering Committee and major stakeholders.

\section{Data analysis and data sharing}

As a healthcare services trial, LOADS' data must be based on clinical records or other hospital data collected by clinical staff as part of treatment of admitted patients, supplemented by administrative data recorded as part of monitoring workplace productivity and safety measures.

LOADS data extraction will commence by clinical administrative staff, who will clean and remove any directly identifiable variables (such as names, dates of birth) of individual patients before making the cleaned dataset available to the PI on a local secure server. Reliance on hospital medical records means reliance on the hospital's procedures for quality control on clinical procedures and recording of events. Although Good Clinical Practice (ICH-GCP) audits or a data monitoring committee are not applicable to a healthcare services study such as LOADS, clinical administrative staff will audit data records for 20 random patient datasets for accuracy before data transfer to the PI. To monitor randomisation during the RCT, the PI will audit a minimum of 1 month's randomisation between RCT study arms and provide feedback to staff on errors in patient allocation.

LOADS data are considered highly sensitive and entitled to a high level of protection by the LDH Privacy Ombudsman, as they are derived directly from records of all acute psychiatric admissions in the LDH catchment area. Requests for deidentified or anonymised data can be made to the contact author and must include a study protocol and detailed data protection plan. Approval is contingent on approval by the LDH Privacy Ombudsman.

\section{Statistical analysis}

Categorical outcomes (nominal, ordinal) may be analysed using binomial regression or chi square analyses. General linear regression or variance models will be used for analyses of continuous variables. If tests or visual inspection indicate the normal distribution assumption is violated, non-parametric tests (such as the Mann-Whitney U test) may be used. Should data indicate the need for modelling, a General linear mixed models or general alinear mixed models paradigm may be applied. Events will be analysed using chi square tables or descriptive analyses only, depending on whether criteria for inferential statistics are satisfied. Analyses will use a 95\% confidence level with any exceptions to this described on a per-analysis basis. Analyses will be conducted using Stata, SPSS and/or $\mathrm{R}$. Software versions that will be described in publications.

In analyses of RCT data, the type of ward/service model will be masked to the statistician during analyses. To prevent early implementation problems such as lack of staff training from influencing the results of the new service model, a 'grace period' of 1 month of data will be exempted from analysis of outcomes. Separate analyses will be conducted for the 12-month RCT and the longitudinal preobservational/postobservational parts of the study. A detailed data analysis plan will be finalised before endpoint analyses commence that will be available to third parties on request to the contact author.

\section{Related or continuing studies}

Separate studies related to implementation of LOADS may be initiated, such as registry-based follow-up of LOADS patients with a parallel comparison to neighbouring hospitals with acute psychiatric wards in Oslo. There are plans for wastewater analysis of in-house substance use and medication concordance, simulation 
training for staff in ODP scenarios and workplace process evaluations.

Force majeure may necessitate modification of elements of LOADS such as practical procedures, study organisation outcomes, or study design. Any exceptions from protocol made on this basis will be described and the integrity of scientific objectives will be discussed.

\section{Ethics and dissemination}

The regional Ethical committee (REC) for South-Eastern Norway has defined LOADS a healthcare services study and granted LOADS exemption from ordinary consent requirements (REC \#29238). The decision was based on LOADS fulfilling strict criteria for exemption including the inclusion of LOADS in Norway's National hospital prioritisation plan, along with appropriate care for participants and the risk of consent causing skewed or minimal recruitment. The protocol is approved by the Scientific Committee of Lovisenberg Diaconal Hospital (LDH), the LDH Privacy Ombudsman as well as the LDH Board of users. The protocol has undergone additional peer review when attracting financial support from the Research Council of Norway and the South-Eastern Norway regional Health Authority.

Dissemination of results is planned for peer-reviewed scientific journals, with follow-up publications in professional journals, popular scientific news sites and journals, and general news media.

Acknowledgements We wish to acknowledge everyone involved in discussing relevant details of the Lovisenberg Open Acute Door Study, including the Lovisenberg Board of Service Users, the Lovisenberg Diaconal Hospital employees and managers, and the 0slo city borough staff and managers.

Contributors NK and HMN designed the study and drafted the initial protocol; NK and HMN applied for funding, NK applied for ethical and regulatory approvals. NK, HMN and A-MI designed systems for collecting patient feedback data. A-MI designed the workplace process evaluation. All authors registered the trial in a clinical registry, contributed in drafting this protocol, approved the final protocol and agreed to be held accountable for all aspects of this article.

Funding This work was supported by public research funding provided by the South Eastern Norway Regional health authority under grant number 26588 and the Research Council of Norway under grant 309 903. Main funding is provided by the Lovisenberg Diaconal Hospital (grant number N/A).

Competing interests None declared.

Patient and public involvement Patients and/or the public were involved in the design, or conduct, or reporting, or dissemination plans of this research. Refer to the Methods section for further details.

Patient consent for publication Not applicable.

Provenance and peer review Not commissioned; externally peer reviewed.

Open access This is an open access article distributed in accordance with the Creative Commons Attribution Non Commercial (CC BY-NC 4.0) license, which permits others to distribute, remix, adapt, build upon this work non-commercially, and license their derivative works on different terms, provided the original work is properly cited, appropriate credit is given, any changes made indicated, and the use is non-commercial. See: http://creativecommons.org/licenses/by-nc/4.0/.

ORCID iD

Nikolaj Kunøe http://orcid.org/0000-0003-4530-2021

\section{REFERENCES}

1 Burns T. Locked doors or therapeutic relationships? Lancet Psychiatry 2016;3:795-6.
2 Mindham RHS. The Retreat at York of 1796 - psychiatry in pictures. Br J Psychiatry 2021;218:286.

3 Funk M, Drew N. WHO QualityRights: transforming mental health services. Lancet Psychiatry 2017;4:826-7.

4 Bhugra D, Tasman A, Pathare S, et al. The WPA-Lancet psychiatry Commission on the future of psychiatry. Lancet Psychiatry 2017;4:775-818.

5 de Jong MH, Kamperman AM, Mulder CL. Open wards in psychiatric clinics and compulsory psychiatric Admissions-Reply. JAMA Psychiatry 2016;73:1293-4.

6 Mezzina R. Community mental health care in trieste and beyond: an "open door-no restraint" system of care for recovery and citizenship. J Nerv Ment Dis 2014;202:440-5.

7 Lang U. Innovative Psychiatrie mit offenen Türen, 2013.

8 Schreiber LK, Metzger FG, Duncker TA, et al. Open doors by fair means: study protocol for a 3-year prospective controlled study with a quasi-experimental design towards (or to implement) an open ward policy in acute care units. BMC Psychiatry 2019;19:149.

9 Bowers L. Safewards: a new model of conflict and containment on psychiatric wards. J Psychiatr Ment Health Nurs 2014;21:499-508.

10 Duxbury J, Baker J, Downe S, et al. Minimising the use of physical restraint in acute mental health services: the outcome of a restraint reduction programme ('REsTRAIN YOURSELF'). Int J Nurs Stud 2019;95:40-8.

11 van Melle AL, Voskes Y, de Vet HCW, et al. High and intensive care in psychiatry: validating the HIC monitor as a tool for assessing the quality of psychiatric intensive care units. Adm Policy Ment Health 2019;46:34-43.

12 Gather J, Henking T, Nossek A. Beneficial coercion in psychiatry? 2017.

13 Otte I, Werning A, Nossek A, et al. Challenges faced by peer support workers during the integration into hospital-based mental health-care teams: results from a qualitative interview study. Int J Soc Psychiatry 2020;66:263-9.

14 Huber CG, Schneeberger AR, Kowalinski E, et al. Suicide risk and absconding in psychiatric hospitals with and without open door policies: a 15 year, observational study. Lancet Psychiatry 2016;3:842-9.

15 Schneeberger AR, Huber CG, Lang UE. Open wards in psychiatric clinics and compulsory psychiatric admissions. JAMA Psychiatry 2016;73:1293.

16 Kowalinski E, Hochstrasser L, Schneeberger AR, et al. [Six years of open-door policy at the University Psychiatric Hospital Basel]. Nervenarzt 2019;90:705-8.

17 Jungfer H-A, Schneeberger AR, Borgwardt S, et al. Reduction of seclusion on a hospital-wide level: successful implementation of a less restrictive policy. J Psychiatr Res 2014;54:94-9.

18 Steinert T, Schreiber L, Metzger FG, et al. Offene Türen in psychiatrischen Kliniken. Nervenarzt 2019;90:680-9.

19 Fan Z, Huang J, Wu Q, et al. Comparison of standard locked-ward treatment versus open-ward rehabilitation treatment for chronic schizophrenic patients. A one-year controlled trial in Canton. Br J Psychiatry Suppl 1994;164:45-51.

20 Smith D, McCauley M. Unlocking an acute psychiatric ward: open doors, absent patients? BJPsych Bull 2018;42:132-3.

21 Beaglehole B, Beveridge J, Campbell-Trotter W, et al. Unlocking an acute psychiatric ward: the impact on unauthorised absences, assaults and seclusions. BJPsych Bull 2017;41:92-6.

22 Mann K, Gröschel S, Singer S, et al. Evaluation of coercive measures in different psychiatric hospitals: the impact of institutional characteristics. BMC Psychiatry 2021;21:1-11.

23 Zwarenstein M, Treweek S, Gagnier JJ, et al. Improving the reporting of pragmatic trials: an extension of the CONSORT statement. BMJ 2008;337:a2390.

24 Chan A-W, Tetzlaff JM, Gøtzsche PC, et al. SPIRIT 2013 explanation and elaboration: guidance for protocols of clinical trials. BMJ 2013;346:e7586.

25 Haugom EW, Granerud A. Shielding in mental health hospitals. Sage Open 2016:6:215824401662952.

26 Hemming K, Haines TP, Chilton PJ, et al. The stepped wedge cluster randomised trial: rationale, design, analysis, and reporting. $B M J$ : 2015;350:h391.

27 Kalagi J, Otte I, Vollmann J, et al. Requirements for the implementation of open door policies in acute psychiatry from a mental health professionals' and patients' view: a qualitative interview study. BMC Psychiatry 2018;18:304.

28 Mental Health Europe. Promising practices in prevention, reduction and elimination of coercion across Europe. Brussels, 2018. Available: https://mhe-sme.org/wp-content/uploads/2019/01/Coercion-Report1.pdf [Accessed 30 Jan 2019]. 
29 Schalast N, Redies M, Collins M, et al. EssenCES, a short questionnaire for assessing the social climate of forensic psychiatric wards. Crim Behav Ment Heal 2008;18:49-58.

30 Nyttingnes $\mathrm{O}$, Rugkåsa J, Holmén $\mathrm{A}$, et al. The development, validation, and feasibility of the experienced coercion scale. Psychol Assess 2017;29:1210-20.

31 Lang UE, Hartmann S, Schulz-Hartmann S, et al. Do locked doors in psychiatric hospitals prevent patients from absconding? Eur J Psychiatry 2010;24:199-204.
32 Hochstrasser L, Fröhlich D, Schneeberger AR, et al. Long-term reduction of seclusion and forced medication on a hospital-wide level: implementation of an open-door policy over 6 years. Eur Psychiatry 2018;48:51-7.

33 Schneeberger AR, Kowalinski E, Fröhlich D, et al. Aggression and violence in psychiatric hospitals with and without open door policies: a 15-year naturalistic observational study. J Psychiatr Res 2017:95:189-95. 